\title{
Extended Gossip Protocol for Diffusion of Multiple Messages and Its Percolation Probability
}

\author{
Tetsuya Ishikawa and Tomohisa Hayakawa \\ Department of Mechanical and Environmental Informatics \\ Tokyo Institute of Technology, Tokyo 152-8552, JAPAN \\ hayakawa@mei.titech.ac.jp
}

\begin{abstract}
Gossip protocol is a randomized broadcast protocol proposed in the field of communication engineering. Gossip protocol is applicable to, for example, a routing protocol on the ad hoc networks. In this paper, we propose an extended gossip protocol which can deal with the multiple messages broadcasting, and analyze the percolation probability of the extended gossip protocol through the percolation theory. In the analysis, we compare 2 cases, one is the case where the source node broadcasts messages in the same time slot, and the other where the source node divides the messages into some groups and broadcasts the messages one after another. Finally, we show some numerical simulation of extended gossip protocol and investigate the property of its percolation probability.
\end{abstract}

\section{Introduction}

Recently, probabilistic broadcast protocol called gossip protocol attracts much attention. Gossip protocol is proposed in the field of communication engineering (e.g., $[1,2])$, and it propagates information by broadcasting a single packet in a probabilistic manner. Gossip protocol is applicable to, for example, a routing protocol on the ad hoc networks as a protocol which is executable on the network whose detailed structure is unknown [3]. As autonomous distributed network has attracted much attention recently, gossip protocol is regarded as an important [4] method to spread the messages across the field, and there are some researches to increase the efficiency of gossip protocol in various ways (e.g., [5-8]). Even though these gossip protocols are designed to broadcast a single message, multiple message cases are not considered explicitly.

In this paper, we propose an extended gossip protocol which can deal with multiple messages broadcasting, and analyze the property of the protocol through the

This research is partially supported by the Aihara Innovative Mathematical Modelling Project, the Japan Society for the Promotion of Science (JSPS) through the Funding Program for World-Leading Innovative R\&D on Science and Technology (FIRST Program), initiated by the Council for Science and Technology Policy (CSTP). percolation theory. The method is predicated on our preceding result for a single massage case [9] and the extended protocol turns out to be more practical than the protocol in [9] in that the case where different kinds of messages would collide can be handled.

The contents of the paper is as follows: In Section 2, some of the basic fact in percolation theory are stated. In Section 3, we define the extended gossip protocol and its critical probability. Finally, we draw conclusions for this paper in Section 4.

\section{Mathematical Preliminaries}

In this section we introduce notation, several definitions, and some key results concerning (site) percolation theory that are necessary for developing the main results of this paper.

\subsection{Percolation Theory and Site Percolation}

Consider a graph $G=(V, E)$, where $V=\left\{v_{1}, v_{2}, \ldots\right\}$ is the collection of the vertices (nodes or sites) and $E=\left\{e_{1}, e_{2}, \ldots\right\}$ is the collection of edges of the graph. Here we assume that the graph $G$ may contain infinite number of vertices. Furthermore, consider the operation of assigning each vertex as open with probability $p$ and as closed with probability $1-p$, where $p \in[0,1]$, independent of the choice for the other vertices. For the vertex $v \in V$, the random variable $X_{v}$ is defined to take 1 if the vertex $v$ is open and 0 if closed. We call the set of connected open sites an open cluster and denote the open cluster containing the vertex $v$ as $C_{v}$. Note that if $v \in V$ and $v^{\prime} \in V$ belong to the same cluster, then $C_{v}=C_{v^{\prime}}$. Furthermore, if $v \in V$ is not open, then $C_{v}=\emptyset$ is assumed. We frequently use $C_{0}$ (Fig. 2.1) throughout the paper, where the vertex 0 is assumed to be the origin of the lattice and belong to $V$.

Let $\Xi$ be the sample space of this operation. For vertices $u_{i} \in V, i=1, \ldots, n$, and the corresponding values $\delta_{i} \in\{0,1\}, i=1, \ldots, n$, of openness/closedness assigned, a subset $A$ of $\Xi$ given by

$$
A=\left\{\xi \in \Xi: X_{u_{1}}(\xi)=\delta_{1}, \ldots, X_{u_{n}}(\xi)=\delta_{n}\right\},
$$

is called a cylindrical set. We denote the number of vertices (resp., open vertices) in $A$ as $\lceil A\rceil$ (resp., $\lfloor A\rfloor$ ). 


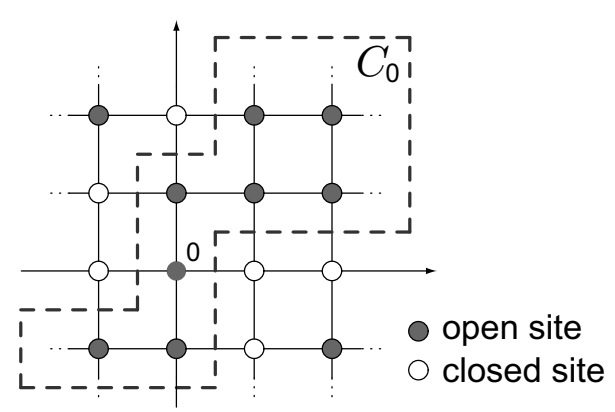

Figure 2.1: An example of $C_{0}$ on the square lattice

In this case, it follows that $\lfloor A\rfloor \triangleq \sum_{i=1}^{\lceil A\rceil} \delta_{i}$, and hence $\lceil A\rceil \geq\lfloor A\rfloor$. Furthermore, let $\mathcal{B}(\Xi)$ be the smallest $\sigma$ algebra generated by the cylindrical sets on $\Xi$, which is assumed to be the $\sigma$-algebra for this operation.

Based on the definitions above, the probability measure for the operation is given by

$$
\mathrm{P}_{p}(A)=p^{\lfloor A\rfloor}(1-p)^{\lceil A\rceil-\lfloor A\rfloor},
$$

on the measurable space $(\Xi, \mathcal{B}(\Xi))$. Note that such a probability measure uniquely exists $[10]$. Henceforth, we consider the probability space give by $\left(\Xi, \mathcal{B}(\Xi), \mathrm{P}_{p}\right)$.

It is important to note that the probability measure $\mathrm{P}_{p}$ has an important property called $F K G$ inequality, that is, if events $A$ and $B$ satisfy $1_{A}(\omega) \leq 1_{A}\left(\omega^{\prime}\right)$ and $1_{B}(\omega) \leq 1_{B}\left(\omega^{\prime}\right)$ for all $\omega, \omega^{\prime} \in \Omega$ such that $\omega \leq \omega^{\prime}$, then

$$
\mathrm{P}_{p}(A \cap B) \geq \mathrm{P}_{p}(A) \mathrm{P}_{p}(B),
$$

where $1_{A}$ denote the indicator function of event $A$ and $\omega \leq \omega^{\prime}$ means $X_{v}(\omega) \leq X_{v}\left(\omega^{\prime}\right), v \in V$. The proof of FKG inequality is found in [11].

\subsection{Critical Probability}

Let $\theta(p)$ be the probability such that the open cluster $C_{0}$ containing the origin has infinite number of vertices; that is,

$$
\theta(p) \triangleq \mathrm{P}_{p}\left(\left|C_{0}\right|=\infty\right),
$$

where $\left|C_{0}\right|$ denotes the cardinal number of the set $C_{0}$. It is important to note that the relationship between $p$ and $\theta(p)$ is numerically calculated to be depicted in the plot given in Fig. 2.2, where there are two phases such that if $p<p_{\mathrm{c}}$ with a threshold $p_{\mathrm{c}} \in(0,1)$, then $\theta(p)=0$ so that the probability of having an infinite cluster is zero. This threshold $p_{\mathrm{c}}$, defined as

$$
p_{\mathrm{c}} \triangleq \sup \{p \in[0,1]: \theta(p)=0\},
$$

is called the critical probability of site percolation and its value depends on the graph $G$ under consideration. Some representative values of the critical probability is given in Table 2.1. Note that the values for the triangle

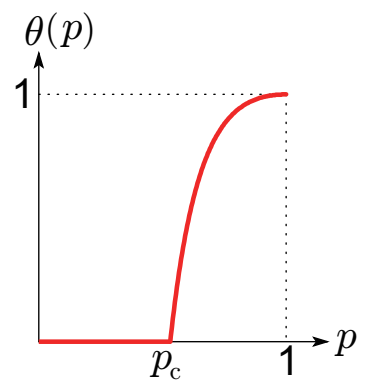

Figure 2.2: Sketch of $p-\theta(p)$ graph

Table 2.1: Critical probabilities of site percolation

\begin{tabular}{cc}
\hline Type of graph & Critical probability \\
\hline Square lattice & 0.593 \\
Triangular lattice & $\frac{1}{2}$ \\
Hexagonal lattice & 0.696 \\
Kagome lattice & $1-2 \sin \frac{\pi}{18}$ \\
Penrose lattice & 0.584 \\
Diamond lattice & 0.428 \\
Body-centered cubic lattice & 0.246 \\
Face-centered cubic lattice & 0.198 \\
\hline
\end{tabular}

and the Kagome lattices are exact through analytical evaluation $[12,13]$.

By contrast, in the case where $G$ is given by the square lattice, it is known that the number of infinite clusters is at most one for any $p>p_{\mathrm{c}}[10]$. Denoting by $N$ the random variable representing the number of infinite clusters, it follows that for $p<p_{\mathrm{c}}$,

$$
\mathrm{P}_{p}(N=k)= \begin{cases}1, & k=0, \\ 0, & \text { otherwise }\end{cases}
$$

and, alternatively for $p>p_{\mathrm{c}}$,

$$
\mathrm{P}_{p}(N=k)= \begin{cases}1, & k=1, \\ 0, & \text { otherwise }\end{cases}
$$

Note that $N \in \mathbb{N} \cup\{\infty\}$.

\section{Extended Gossip Protocol}

In this section, we first describe the extended gossip protocol proposed in this paper. Next, we give a mathematical representation of the extended gossip protocol on 2-dimensional square lattice with 2 messages and discuss the notable properties of the protocol.

\subsection{Description of Extended Gossip Protocol}

The extended gossip protocol used in this paper is predicated on the conventional gossip protocol proposed by Hass et al. [3], and we give additional rules to it. Most important point of the additional rule is the management of multiple messages. If multiple messages are broadcasted from the neighbor of a node, the node may 
receive different messages at the same time slot. Here we assume that any node can deal with multiple messages at the same time slot because, in wireless sensor networks which gossip protocol is likely to applied, each node should have low performance. As such, we consider the case if multiple messages are broadcasted from the neighbor of a node at the same time slot, then the node actually select and receive one of these messages randomly.

In this paper, the extended gossip protocol on the graph $G=(V, E)$ is implemented by following rules:

Rule 1: There is only one source node in the network and all the other nodes do not have messages to be spread. (Without loss of generality, the source node is placed at the origin 0.) At the first step, the source node broadcasts the message with probability 1 .

Rule 2: If a neighbor node of $v \in V$ broadcasts a message, then node $v$ can receive it. A node other than origin cannot deal with multiple messages at the same time slot, so that if multiple messages are sent from a neighbor of $v$, then $v$ randomly select and receive only one of them with uniform distribution.

Rule 3: A node which receive a message broadcasts the message in the next step with probability $q$, and it does not with probability $1-q$.

Rule 4: Once a node receive a message, it dose not receive the same message again (so that it dose not broadcast the same message more than once).

The probability $q$ in Rule 3 is called gossip probability. Rules 1, 3, and 4 are the same as in the protocol used in [3] and [9], while the treatment of multiple messages in Rule 2 is original in this framework.

\subsection{Mathematical Representation of Extended Gossip Protocol with Two Messages}

Now, we give a mathematical representation of extended gossip protocol with 2 messages. In the following discussion, we restrict the network structure to 2dimensional square lattice $\mathbb{L}^{2}=\left(V_{\mathrm{L}}, E_{\mathrm{L}}\right)$.

Let $q \in[0,1]$ be the gossip probability and consider the case where source node broadcasts 2 messages $m_{1}$ and $m_{2}$ in this order. Furthermore, let $\left(\Omega, \mathcal{F}, \mathrm{P}_{q}\right)$ be the probability space associated with the extended gossip protocol and let $X_{1}^{v}: \Omega \rightarrow\{0,1\}$ is a random variable which represents the case where the node $v$ broadcasts the message $m_{1}$ or not at the time the node $v$ receive $m_{1}$ from its neighbor. If the node $v$ broadcasts the message $m_{1}$ when the node $v$ receive it, then $X_{1}^{v}=1$. Likewise, let $X_{2}^{v}: \Omega \rightarrow\{0,1\}$ be a random variable for the message $m_{2}$. Let $X_{\times}^{v}: \Omega \rightarrow\left\{m_{1}, m_{2}\right\}$ be a random variable which represent that which message is received if $m_{1}$ and $m_{2}$ are broadcasted from the neighbor of $v$ at the same time slot. For example, if $X_{1}^{v}=1$ and $X_{\times}^{v}=m_{1}$, then the node $v$ select and receive the message $m_{1}$ and broadcasts $m_{1}$ in the next step.

Next, let $C_{1}, C_{2}, F_{1}^{k}, F_{2}^{k}$ be random variables which take values in $2^{V_{\mathrm{L}}}$ defined as

$$
\begin{aligned}
& C_{1}(\omega) \triangleq \mathcal{Z}_{0}\left(\left\{v \in V_{\mathrm{L}}: X_{1}^{v}(\omega)=1\right\}\right), \\
& C_{2}(\omega) \triangleq \mathcal{Z}_{0}\left(\left\{v \in V_{\mathrm{L}}: X_{2}^{v}(\omega)=1\right\}\right), \\
& F_{1}^{k}(\omega) \triangleq\left\{v \in V_{\mathrm{L}}: \operatorname{dist}_{C_{1}(\omega)}(v)-\operatorname{dist}_{C_{2}(\omega)}(v)=k,\right. \\
& \left.X_{\times}^{v}(\omega) \neq m_{1}\right\}, \\
& F_{2}^{k}(\omega) \triangleq\left\{v \in V_{\mathrm{L}}: \operatorname{dist}_{C_{1}(\omega)}(v)-\operatorname{dist}_{C_{2}(\omega)}(v)=k,\right. \\
& \left.X_{\times}^{v}(\omega) \neq m_{2}\right\}, \\
& F^{k}(\omega) \triangleq F_{1}^{k}(\omega) \cup F_{2}^{k}(\omega), \\
& D^{k}(\omega) \triangleq \mathcal{Z}_{0}\left(C_{1}(\omega) \backslash F_{1}^{k}(\omega)\right) \cap \mathcal{Z}_{0}\left(C_{2}(\omega) \backslash F_{2}^{k}(\omega)\right),
\end{aligned}
$$

where $\mathcal{Z}_{v}(U)$ denote the connected component of $U \subset$ $V_{\mathrm{L}}$ containing $v \in V_{\mathrm{L}}$ and such that if $v \notin U$, then $\mathcal{Z}_{v}(U)$ is defined as $\emptyset$. Also, $\operatorname{dist}_{U}(v)$ denote the graph distance on $U$ between $v$ and the origin.

The random variables $C_{i}, i=1,2$, represent the set of nodes which can receive the message $m_{i}$ in the case where there is no message collision. Furthermore, the random variables $F_{i}^{k}, i=1,2$, are the set of nodes in which message $m_{i}$ can be discarded by the message collision due to the fact that the temporal difference of $m_{1}$ and $m_{2}$ being $k$ implies that the distance of the nodes of the messages $m_{1}$ and $m_{2}$ which collide is $k$. Note that $F_{1}^{k}$ and $F_{1}^{k}$ satisfy $F_{1}^{k}(\omega) \cap F_{2}^{k}(\omega)=\emptyset, \omega \in \Omega$. In addition, the random variable $F^{k}$ represents the set of nodes in which the message collision occurs and and the random variable $D^{k}$ represents the set of nodes which can receive both messages $m_{1}$ and $m_{2}$.

Let the probability measure $\mathrm{P}_{q}$ satisfy the following conditions:

- $\mathrm{P}_{q}\left(X_{1}^{v}=1\right)=q, \mathrm{P}_{q}\left(X_{2}^{v}=1\right)=q$,

- $\mathrm{P}_{q}\left(X_{\times}^{v}=m_{1}\right)=\frac{1}{2}$,

- $X_{1}^{v}$ and $X_{2}^{v}$ are independent, $v \in V_{\mathrm{L}}$,

- $X_{1}^{u}$ and $X_{1}^{v}$ are independent, $u, v \in V_{\mathrm{L}}$,

- $X_{2}^{u}$ and $X_{2}^{v}$ are independent, $u, v \in V_{\mathrm{L}}$,

- $X_{\times}^{u}$ and $X_{\times}^{v}$ are independent, $u, v \in V_{\mathrm{L}}$.

Now, we define the percolation probability of the extended gossip protocol using the above notations as follows: 

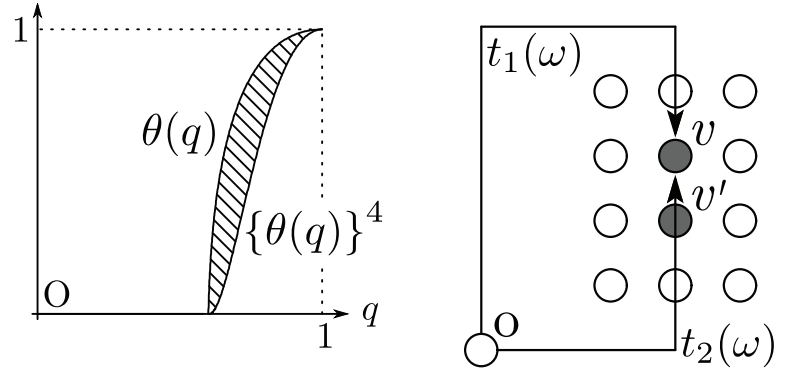

Figure 3.1: $\eta^{\infty}(q)$ and $\theta(q)$

Figure 3.2: Example of $v^{\prime}$

Definition 3.1. Consider the extended gossip protocol on the square lattice $\mathbb{L}^{2}$ with 2 messages $m_{1}$ and $m_{2}$ and let $k$ be the temporal difference of the two messages $m_{1}$ and $m_{2}$ sent at the origin. The percolation probability $\eta^{k}(q)$ of the extended gossip protocol is defined as

$$
\eta^{k}(q) \triangleq \mathrm{P}_{q}\left(\rho\left(D^{k}\right)=\infty\right)
$$

where

$$
\rho(U) \triangleq \max \left\{\operatorname{dist}_{V_{\mathrm{L}}}(v): v \in U\right\},
$$

and $U \subset V_{\mathrm{L}}$. Furthermore, the value $q_{\mathrm{c}}^{k}$ given by

$$
q_{\mathrm{c}}^{k} \triangleq \inf \left\{q \in[0,1]: \eta^{k}(q)>0\right\} .
$$

is called critical probability of the extended gossip protocol.

\subsection{Some Properties of Extended Gossip Protocol and Its Percolation Probability}

First, we discuss the case where the temporal distance of messages $m_{1}$ and $m_{2}$ sent at the origin is infinity.

Theorem 3.1. Let $\theta(p)$ be the critical probability of site percolation on the 2-dimensional square lattice and let $\eta^{k}(q)$ be the percolation probability of the extended gossip protocol on the 2-dimensional square lattice with 2 messages where $k$ is the temporal distance of messages sent at the origin. The percolation probability $\eta^{\infty}(q)$ satisfies

$$
\{\theta(q)\}^{4} \leq \eta^{\infty}(q) \leq \theta(q), q \in[0,1] .
$$

Theorem 3.1 states the lower and upper bound of percolation probability of the extended gossip protocol in the case where no message collision occurs. In other words, the graph of $\eta^{\infty}(q)$ is in the oblique lined area of Figure 3.1. Hence, we immediately conclude that the critical probability of the extended gossip protocol on $\mathbb{L}^{2}$ with $k \rightarrow \infty$ is identical to the critical probability of site percolation on $\mathbb{L}^{2}$.

Next, we discuss the case where the temporal distance of messages $m_{1}$ and $m_{2}$ is finite. We present the 2 lemmas below that are used in the proof of the next theorem.
Lemma 3.1. If $q \in[0,1)$, then $\mathrm{P}_{q}\left(\left|F^{k}\right|=\infty\right)=0$.

Lemma 3.2. If $\left|F^{k}(\omega)\right|<M$ for a $\omega \in \Omega$, then there exists $\omega^{\prime \prime} \in \Omega$ such that

- if $\rho\left(D^{k}(\omega)\right)=\infty$ then $\rho\left(D^{k+2}\left(\omega^{\prime \prime}\right)\right)=\infty$,

- $\alpha \mathrm{P}_{q}(\{\omega\}) \leq \mathrm{P}_{q}\left(\left\{\omega^{\prime \prime}\right\}\right) \leq \beta \mathrm{P}_{q}(\{\omega\})$,

where

$$
\begin{aligned}
& \alpha \triangleq \min \left\{\left(\frac{1-q}{q}\right)^{N},\left(\frac{q}{1-q}\right)^{N}\right\}, \\
& \beta \triangleq \max \left\{\left(\frac{1-q}{q}\right)^{N},\left(\frac{q}{1-q}\right)^{N}\right\},
\end{aligned}
$$

and $N$ is an integer.

Theorem 3.2. Let the temporal distance $k$ be a finite integer. Then the inequality $q_{\mathrm{c}}^{k} \geq q_{\mathrm{c}}^{k+2}$ holds.

Theorem 3.2 implies that the larger the critical probability of the extended gossip protocol is the smaller the temporal difference $k$ becomes. Note that Theorem 3.2 compares the temporal difference between $k$ and $k+2$, not $k$ and $k+1$. In fact, in the case where the graph is the 2-dimensional square lattice, the critical probability of the extended gossip protocol satisfies

$$
\begin{aligned}
& q_{\mathrm{c}}^{0} \geq q_{\mathrm{c}}^{2} \geq q_{\mathrm{c}}^{4} \geq \cdots \geq q_{\mathrm{c}}^{\infty}, \\
& q_{\mathrm{c}}^{1}=q_{\mathrm{c}}^{3}=q_{\mathrm{c}}^{5}=\cdots=q_{\mathrm{c}}^{\infty} .
\end{aligned}
$$

This implies that if the temporal difference $k$ is odd, then no message collision occurs and (10) holds. Inequality (9) is a result of Theorem 3.2.

\section{Conclusion}

In this paper, we proposed the extended gossip protocol to be able to manage the multiple messages based on gossip protocol. Furthermore, we analyze the percolation probability of the extended gossip protocol. Simulation result in Section 3 implies that the inequality $\eta^{0}(q) \leq \eta^{2}(q)$ holds for all $q \in[0,1]$, but we can prove only $q_{\mathrm{c}}^{0} \geq q_{\mathrm{c}}^{2}$ which implies that the inequality $\eta^{0}(q) \leq \eta^{2}(q)$ holds in the neighborhood of the critical probability. If the inequality is shown for all $q$, we will propose theoretically the optimal broadcasting procedure of the multiple messages.

\section{References}

[1] B. Williams and T. Camp, "Comparison of broadcasting techniques for mobile ad hoc networks," in Proc. ACM Sympo. Mobile Ad Hoc Netw. Compu., (Lausanne, Switzerland), pp. 194-205, June 2002.

[2] Y.-C. Tseng, S.-Y. Ni, Y.-S. Chen, and J.-P. Sheu, "The broadcast storm problem in a mobile ad hoc network," Wirel. Netw., vol. 8, pp. 153-167, 2002. 
[3] Z. J. Haas, J. Y. Halpern, and L. Li, "Gossip-based ad hoc routing," IEEE/ACM Trans. Netw., vol. 14, pp. 479-491, 2006.

[4] I. F. Akyildiz, W. Su, Y. Sankarasubramaniam, and E. Cayirci, "Wireless sensor networks: a survey," Compu. Netw., vol. 38, no. 4, pp. 393 - 422, 2002.

[5] Y. Sasson, D. Cavin, and A. Schiper, "Probabilistic broadcast for flooding in wireless mobile ad hoc networks," in Proc. IEEE Wirel. Commu. Netwo. Conf., (New Orleans, LA), pp. 1124-1130, March 2003.

[6] M. Burmester, T. V. Le, and A. Yasinsac, "Adaptive gossip protocols: Managing security and redundancy in dense ad hoc networks," Ad Hoc Netw., vol. 5, pp. 313323, 2007.

[7] M. G. Rabbat, "On spatial gossip algorithms for average consensus," in Proc. IEEE Stat. Signal Process. Workshop, pp. 705-709, August 2007.

[8] R. Beraldi, "The polarized gossip protocol for path discovery in MANETs," Ad Hoc Netw., vol. 6, pp. 79-91, 2008.

[9] T. Ishikawa and T. Hayakawa, "On equivalence of critical probabilities of dynamic gossip protocol," SICE J. Contr. Meas. Sys. Integr., vol. 4, no. 3, pp. 254-259, 2011.

[10] B. Bollobas and O. Riordan, Percolation. Cambridge, UK: Cambridge University Press, 2006.

[11] G. Grimmett, Percolation. New York, NY: SpringerVerlag, 1999.

[12] H. Kesten, "The critical probability of bond percolation on the square lattice equals $1 / 2$," Communications in Mathematical Physics, vol. 74, pp. 41-59, 1980.

[13] J. C. Wierman, "Bond percolation on honeycomb and triangular lattices," Adv. Appl. Prob., vol. 13, pp. 298313, 1981. 